\title{
Emerging transcatheter options for tricuspid regurgitation: Many shades of gray
}

\author{
Vinayak Bapat, MBBS, MS, MCh, DNB (Surg), DNB (Card Surg), FCRSEd, FRCSCTh, ${ }^{\mathrm{a}}$ and \\ Gilbert H. L. Tang, MD, MSc, MBA ${ }^{\text {b }}$
}

Feature Editor's Note-In this edition of the Journal, Drs Bapat and Tang provide a broad view of the current state of transcatheter therapies for the treatment of tricuspid regurgitation. It is perhaps a far more complex subject than transcatheter therapies for other cardiac valves. This is because the tricuspid is the valve least understood and with which surgeons have the least experience, as evidenced by its various monikers "the forgotten valve," "the enigmatic valve," and "the untreated valve." This applies not only to its anatomic relationship to structures such as the coronary sinus, aortic valve, and conductions system; paucity of experience with TR correction also means that there are knowledge gaps in the effect of $T R$ reduction or elimination on right ventricular function and remodeling, to say nothing of the role of pulmonary hypertension. Compound this with the fact that the transcatheter valvular therapies were originally designed for left-sided valves. Adoption and adaptation of these devices for the treatment of TR may seem the most logical way to proceed, but as Dr Bapat's article reveals, much work remains before clinical efficacy and benefit appear on the horizon.

\section{Dawn S. Hui, MD, and Richard Lee, MD, MBA}

Severe tricuspid regurgitation (TR) is a common valvular heart disease, with a reported prevalence of approximately $4 \%$ in the adult population. ${ }^{1}$ Among patients with severe TR, approximately $90 \%$ have a secondary etiology. ${ }^{2}$ Whereas functional mitral regurgitation is primarily explained by the displacement of the papillary muscles related to left ventricular dilation, distension of the annulus is by far the dominant mechanism causing secondary TR. ${ }^{3}$ Isolated severe TR has been identified as an independent predictor of death and heart failure hospitalization, and the prognosis

\footnotetext{
From the ${ }^{a}$ Division of Cardiac Surgery, Columbia University Medical Center and ${ }^{\mathrm{b}}$ Department of Cardiovascular Surgery, Mount Sinai Health System, New York, NY.

Received for publication Oct 20, 2019; revisions received Jan 8, 2020; accepted for publication Jan 14, 2020; available ahead of print April 1, 2020.

Address for reprints: Vinayak Bapat, MBBS, MS, MCh, DNB (Surg), DNB (Card Surg), FCRSEd, FRCSCTh, Division of Cardiac Surgery, Columbia University Medical Center, 177 Fort Washington Ave, Floor MHB 7-435, New York, NY 10032 (E-mail: vnbapat@yahoo.com).

J Thorac Cardiovasc Surg 2020;160:1460-4 $0022-5223 / \$ 36.00$

Copyright $₫ 2020$ Published by Elsevier Inc. on behalf of The American Association for Thoracic Surgery

https://doi.org/10.1016/j.jtcvs.2020.01.102
}

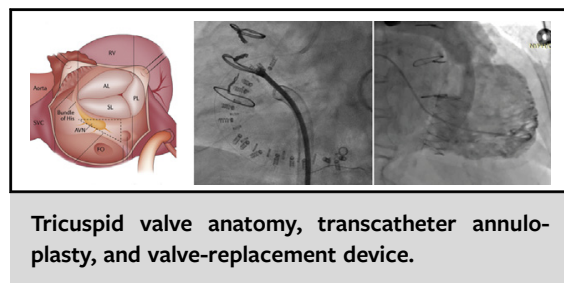

\begin{abstract}
CENTRAL MESSAGE
Transcatheter options are being explored as an option to treat severe TR. Although early studies have shown some promise, considerable hurdles remain with respect to patient selection, imaging, procedure, and data interpretation.
\end{abstract}

This Invited Expert Opinion provides a perspec tive on the following paper: J Am Coll Cardiol. 2019;73:1905-1915. 2019.01.062.

See Commentary on page 1465.

of untreated TR remains poor. ${ }^{4}$ Owing to the elevated mortality associated with tricuspid surgery, particularly following previous left-sided valve surgery or tricuspid repair surgery, there may be reluctance to perform isolated tricuspid surgery. ${ }^{5,6}$ This explains only 5005 isolated tricuspid valve (TV) procedures reported in a large contemporary US nationwide registry over a decade, with mortality reported ranging from $8.8 \%$ to $9.7 \% .{ }^{6,7}$ Hence, it is not surprising that there is an emergence of transcatheter treatment options to address this unmet clinical need, to reduce mortality and morbidity associated with conventional treatment.

However, transcatheter tricuspid therapy has several potential pitfalls that need to be overcome and its effectiveness proven if the therapy were to be offered to a significant number of patients. These are (1) anatomic challenges, (2) physiological challenges, (3) intraprocedural imaging, (4) presence of pacing or other leads, (5) device options, and (6) efficacy and building evidence. 


\section{ANATOMIC CHALLENGES}

Three important anatomic structures are at risk of injury or impingement during TV intervention: (1) the conduction system, (2) aortic valve, and (3) right coronary artery ${ }^{8}$ (Figure 1, A). The relationship between the first 2 is fairly constant but right coronary artery relationship varies and can affect annular-based repair devices. Further, fragile tissue quality of tricuspid leaflets and annulus, thin right ventricle, and angulation to access TV through transvenous access add another layer of challenge to the intervention procedures. Advances in imaging techniques including an effort to standardize "interventional tricuspid anatomy" along with better delivery systems will play a critical role in avoiding complications and determining the success of these treatments. ${ }^{9,10}$

\section{PHYSIOLOGICAL CHALLENGE}

A majority of the patients with TR are managed with medical therapy, which often leads to a significant delay in referral for intervention. It is not uncommon to encounter a patient with end-stage right ventricular (RV) failure, extreme annular dilatation, and severe leaflet tethering in whom any form of intervention is likely futile. ${ }^{5}$ Identifying this patient subset is important. Furthermore, during early experience, a discrepancy was observed that reduction in effective regurgitant orifice area with clinical benefit and traditional grading of TR. ${ }^{11-13}$ Hence, a new TR grading system has been proposed to understand the impact of intervention and to guide better patient selection. ${ }^{14}$ Unlike surgery, where the goal is to eliminate TR, catheter interventions other than transcatheter tricuspid valve replacement (TTVR) reduce but not eliminate TR. Current research is focused on understanding the intricate balance and interaction among TR severity, RV function, and pulmonary hypertension and its combined impact on choosing a particular treatment strategy, ie, reduction versus elimination of $\mathrm{TR}^{8,15}$

\section{INTRAPROCEDURAL IMAGING}

While cardiac computed tomography is critical in case planning, transesophageal echo (TEE) forms the intraprocedural backbone of TV interventions. TEE imaging can be inadequate, as TV is further away from the probe, the TV apparatus is more complex and varied, and it is also influenced by enlargement of the right atrium and ventricle. Furthermore, the presence of other prosthetic devices, including valves, rings, and pacing leads, add to imaging challenges. Four-dimensional intracardiac echocardiography may provide a complementary imaging modality to TEE in optimizing intraprocedural guidance to TV interventions. ${ }^{16}$

\section{PRESENCE OF LEADS CROSSING THE TV}

It is a well-known fact that the presence of implantable cardiac deices, especially pacemakers and defibrillators, may cause or worsen TR. ${ }^{17}$ The mechanism may be mechanical perforation or laceration of the leaflets, scarring and/or restriction of the leaflets, or asynchronized activation of the right ventricle. Prevalence of TR varies between $25 \%$ and $29 \%$ and new TR may appear as late as a few years after implantation. ${ }^{17}$ The presence of leads may limit the use of transcatheter TV treatment and was a contraindication in a few trials. With increasing experience, TTVR devices and repair technologies such as MitraClip, FORMA, and TriCinch have treated few carefully selected patients with TR in the presence of leads. Further experience is needed

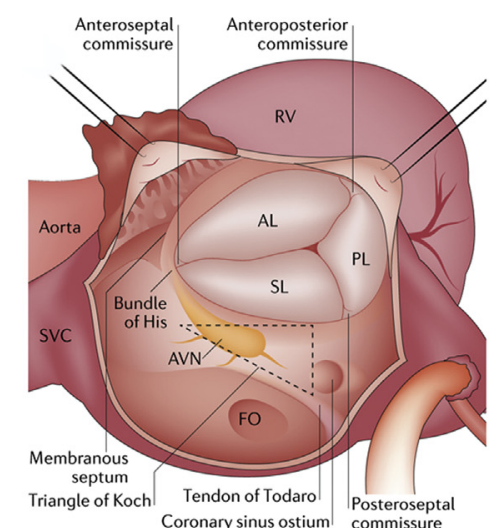

A

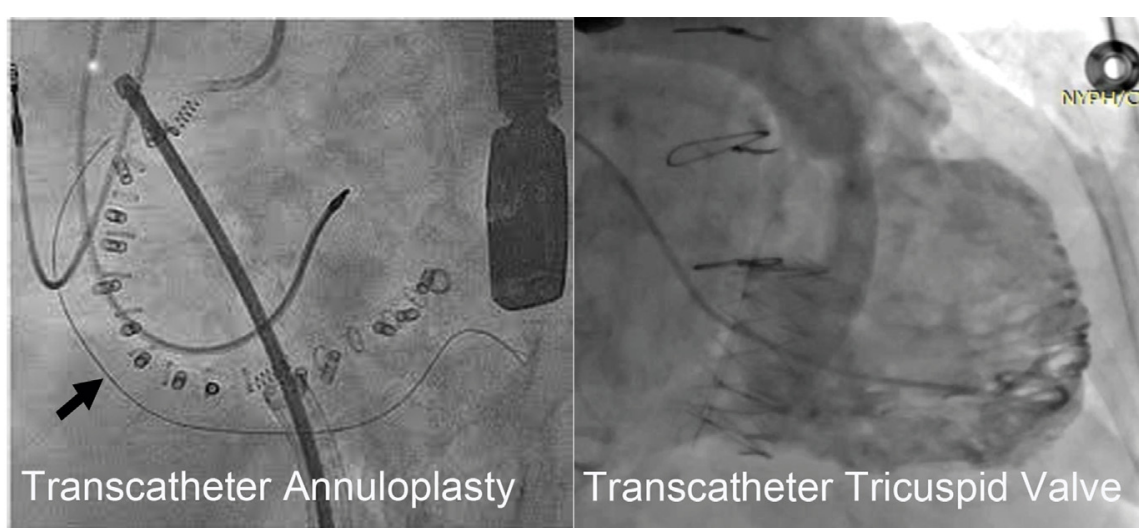

B
C

FIGURE 1. Tricuspid valve anatomy and transcatheter options. A, Tricuspid valve anatomy, demonstrating 3-leaflet structure and surrounding anatomy. B, Cardioband annuloplasty device after implantation and cinching with coronary wire seen in the right coronary artery (arrow). C, Navigate tricuspid valve replacement device after implantation with right ventriculogram demonstrating competent valve. $R V$, Right ventricle; $A L$, anterior leaflet of tricuspid valve; $P L$, posterior leaflet of tricuspid valve; $S L$, septal leaflet of tricuspid valve; $S V C$, superior vena cava; $A V N$, atrioventricular node; $F O$, fossa ovalis. 
to understand the interaction and ability of these devices to work efficiently in the presence of leads.

\section{DEVICE OPTIONS}

Transcatheter TV interventions try to mimic surgical options. Transcatheter repair options are targeted to achieve reduction in TR by either modifying annular geometry (direct or indirect) or by closing the leaflet gaps (direct or indirect). Tricuspid replacement could be either orthotopic or heterotopic replacement ${ }^{8}$ (Table 1). Some of the interventional approaches such as FORMA (Edwards Lifesciences LLC, Irvine, Calif) and heterotopic valve replacement are usually undertaken as the "last resort" and are focused on palliation rather than cure. ${ }^{8}$ Replacement option may in addition have specific challenges such as large device and delivery system size and need for anticoagulation. ${ }^{18}$

\section{EFFICACY AND EVIDENCE Edge-to-Edge Repair}

The MitraClip (Abbott Structural Heart, Santa Clara, Calif) remains the most common off-label procedure to repair severe TR. Originally designed for the treatment of mitral regurgitation, the MitraClip delivery technique was modified to grasp TV leaflets to reduce TR. More than 1000 cases have been performed worldwide, and the most recent TRIVALVE registry reported favorable outcomes. ${ }^{19}$ However, the edge-to-edge technique faces several challenges when applied to TV. Imaging leaflet coaptation gap remains the single most important independent predictor of procedural success. Steering of the delivery catheter can be suboptimal due to the fact that the device was not designed for the TV, and large coaptation gaps in addition to the delicate nature of TV increase risk of leaflet tear and procedural failure. Bicuspidization achieved by grasping anterior and septal leaflets has shown more favorable outcomes and the zipping technique by using more than one clip appears to yield a more reliable result. In a multicenter registry reported by Nickenig and colleagues, ${ }^{20}$ 64 patients underwent edge-to-edge repair (functional TR, $88 \%$ ) with reported procedural success of $97 \%,>2$ clips were used in $50 \%$ of patients with $>1$ TR grade reduction. In-hospital death was 5\%. Most reported improved functional status at 30-day follow-up. ${ }^{20}$

The ongoing TRILUMINATE (Trial to Evaluate Treatment With Abbott Transcatheter Clip Repair System in Patients With Moderate or Greater Tricuspid Regurgitation) early feasibility study (prospective, single-arm, multicenter study, conducted in 21 centers across Europe and the United States) to assess effectiveness (defined as TR reduction of at least grade 1 at 30 days) and safety (composite of major adverse events) showed promising 6-month results in 85 patients with significant TR reduction (TR reduction of at least more than 1 grade achieved in $87 \%$, proportion of subjects with moderate or less TR increased from $6 \%$ at baseline to $57 \%$ at 6 months), annular reduction and positive RV modeling (Transcatheter Cardiovascular Therapeutics [TCT] meeting, 2019). A majority of patients had $>1$ clip $(80 \%)$ and implant success was $100 \%$. At 6 months $3.7 \%$ experienced major adverse event $(2=$ death, $1=$ renal failure). Five patients experience clip detachment without embolization or adverse clinical event. Of note, $29 \%$ patients had massive TR, and 37\% had torrential TR before treatment versus only $7 \%$ with similar degree of residual TR after the TriClip procedure. This makes one think that earlier intervention may reduce technical challenges and achieve a more robust reduction of TR.

TABLE 1. List of transcatheter devices with first-in-human experience

\begin{tabular}{lllll}
\hline Procedure & Structure targeted & Device name & \multicolumn{1}{c}{ Mechanism } & Comment \\
\hline Repair & Annulus & Cardioband & Partial band & Posterior annuloplasty \\
& & Cillipede IRIS & Complete ring & Suture annuloplasty \\
& & MIA & Bicuspidization & Withdrawn from market \\
& Leaflet & TriCinch & Annular reshaping & Withdrawn from market \\
& & Trialign & Bicuspidization & \\
Replacement & MitraClip & Clipping adjacent leaflets & Clipping adjacent leaflets and a spacer \\
& Orthotopic & PASCAL & Spacer anchored in RV & Tynes engaged with chordae \\
& & FORMA & Cork shape and tynes & \\
& Gate & Antrepid & & \\
& Heterotopic & Evoque & in IVC/SVC & \\
\hline
\end{tabular}

$R V$, Right ventricle; $I V C$, inferior vena cava; $S V C$, superior vena cava. 
Newer device iterations, such as longer and larger clips to increase leaflet coaptation after grasping, more flexible delivery systems, and the introduction of devices with similar mechanism (PASCAL; Edwards Lifesciences LLC), may help achieve better outcomes in these patients. ${ }^{20,21}$

\section{Annuloplasty Devices}

The Cardioband (Edwards Lifesciences LLC) is a flexible annuloplasty band designed to mimic surgical TV repair and reduce TR (Figure 1, B). The device is CE marked and the US early feasibility study is underway. Nickenig and colleagues ${ }^{22}$ reported 6-months outcome with Cardioband system in 30 patients enrolled in a prospective, single-arm, multicenter European study. TRI-REPAIR study (Tricuspid Regurgitation RePAir With Cardioband Transcatheter System) included patients with symptomatic moderate-to-severe TR in absence of leftsided disease who were deemed inoperable by the local heart team. ${ }^{22}$ Mean age was 75 years, $73 \%$ were female, $36.6 \%$ had previous open-heart surgery, $52 \%$ had massive or torrential TR, $83 \%$ were in New York Heart Association class III/IV at baseline, and mean ejection fraction was $58 \%$. Society of Thoracic Surgeons score was $2.6 \%$ and European System for Cardiac Operative Risk Evaluation II was $4.1 \%$. Technical success was $100 \%$. Between baseline and 6 months, the average reduction in the annular septolateral diameter was $9 \%$, with an effective regurgitant orifice area of $50 \%$. At 30 days, 2 patients died, 4 had bleeding complications, 3 had coronary complications, and 1 had conduction disturbance. Although the authors concluded that implantation of Cardioband is feasible, safe, and effective, there was only modest reduction in the TR reduction. The authors also acknowledged the timeconsuming aspect of the procedure and challenges in patient screening with regards to right coronary artery proximity. Results from early feasibility study in the United States (22 patients) presented at the TCT meeting (San Francisco, October 2019) showed excellent safety profile $(95.5 \%$ technical success), sustained TR, and annular reduction at 30 days. Anatomic limitations, dependency of intraprocedural imaging, and need for multiple anchors (increasing the risk of bleeding and damage to surrounding structures) remain key hurdles for this technology to become mainstream. Other annuloplasty concepts such as MIA (Micro Interventional devices, Newtown, Pa) and Millipede IRIS (Boston Scientific, Santa Rosa, CA) have limited experience, and further studies need to be conducted to understand their early-in-human results. ${ }^{8}$

\section{Valve Replacement}

TTVR in native TR has been performed with the Gate system (Navigate, Cleveland, Ohio) via a direct transatrial or transjugular venous approach (Figure 1,C). Compassionate use experience in 35 patients showed a 30-day mortality rate of $13.8 \%$ due to comorbidities and advanced RV dysfunction in these patients. Presence of pacemaker may not be a contraindication for TTVR. ${ }^{17}$

Transfemoral access with Intrepid (Medtronic, Minneapolis, Minn) and Evoque (Edwards Lifesciences LLC) TTVR devices have also been performed, showing early promise. The main challenge for TTVR is the device size required to address large annuli, need for anticoagulation, and understanding which patients will not tolerate elimination of TR.

\section{Other Technologies}

Other technologies with out-of-box concepts such as 4-Tech annular modification and heterotopic valve implantation has limited experience at this stage. Also, certain technologies such as FORMA (Edwards Lifesciences, LLC) and Trialign (Mitralign Inc, Boston, Mass) have been withdrawn due to safety and efficacy concerns. ${ }^{11,23-25}$

In the absence of good surgical options, transcatheter treatment may emerge as a good alternative in patients with isolated TV disease with unmet clinical need. Although in infancy, early experience in high-risk patients is encouraging. Multiple devices have been introduced and caution is mandatory, as a few devices have been withdrawn due to concerns of safety and/or efficacy. Hence, improved patient selection, improved device iteration, earlier intervention, standardizing TR definitions, optimizing intraprocedural imaging, and evaluating impact on mid-term and long-term outcomes will define the role of these novel treatments. More importantly, a greater understanding will also be needed in choosing appropriate device for the appropriate anatomy and physiology to achieve a durable result.

\section{Conflict of Interest Statement}

Dr Bapat is consultant for Medtronic, Inc, Boston Scientific, and 4Tech. Dr Tang is a consultant for Abbott Structural Heart.

\section{References}

1. Nath J, Foster E, Heidenreich PA. Impact of tricuspid regurgitation on long-term survival. J Am Coll Cardiol. 2004:43:405-9.

2. Mutlak D, Lessick J, Reisner SA, Aronson D, Dabbah S, Agmon Y. Echocardiography-based spectrum of severe tricuspid regurgitation: the frequency of apparently idiopathic tricuspid regurgitation. J Am Soc Echocardiogr. 2007;20: 405-8.

3. Dreyfus GD, Martin RP, Chan KM, Dulguerov F, Alexandrescu C. Functional tricuspid regurgitation: a need to revise our understanding. J Am Coll Cardiol. 2015;65:2331-6.

4. Topilsky Y, Nkomo VT, Vatury O, Michelena HI, Letourneau T, Suri RM, et al. Clinical outcome of isolated tricuspid regurgitation. JACC Cardiovasc Imaging. 2014; 7:1185-94.

5. Kim YJ, Kwon DA, Kim HK, Park JS, Hahn S, Kim KH, et al. Determinants of surgical outcomes in patients with isolated tricuspid regurgitation. Circulation. 2009; 120:1672-8.

6. Zack CJ, Fender EA, Chandrashekar P, Reddy YNV, Bennett CE, Stulak JM, et al. National trends and outcomes in isolated tricuspid valve surgery. $J$ Am Coll Cardiol. 2017;70:2953-60. 
7. Alqahtani F, Berzingi CO, Ajohani S, Hizazi M, Al-Hallak A, Alkhouli M Contemporary trends in the use and outcomes of surgical treatment of tricuspid regurgitation. J Am Heart Assoc. 2017;6:e007597.

8. Asmarats L, Puri R, Latib A, Navia J, Rodes-Cabau J. Transcatheter tricuspid in terventions: landscapes, challenges and future directions. J Am Coll Cardiol. 2018:25:2935-56.

9. Russo M, Kuwata S, Pozzoli A, Maisano F, Taramasso M. Understanding the tricuspid valve for transcatheter valve repair: comparative anatomy of different imaging modalities. Eur Heart J Cardiovasc Imaging. 2017;18: 823.

10. Hahn RT. State of art review of echocardiographic imaging in the evaluation and treatment of functional tricuspid regurgitation. Circ Cardiovasc Imaging. 2016;9: $\mathrm{e} 005332$.

11. Schofer J, Bijuklic K, Tiburtius C, Hansen L, Groothuis A, Hahn RT. First-inhuman transcatheter tricuspid valve repair in a patient with severely regurgitant tricuspid valve. J Am Coll Cardiol. 2015;65:1190-5.

12. Latib A, Agricola E, Pozzoli A, Denti P, Taramasso M, Spagnolo P, et al. First-inman implantation of a tricuspid annular remodeling device for functional tricuspid regurgitation. JACC Cardiovasc Interv. 2015;8:e211-4.

13. Malasa M, Werner N, Nickenig G, Hammerstingl C. Transcatheter tricuspid valve repair in a patient with isolated functional tricuspid valve regurgitation. Eur Heart J. 2016;37:855.

14. Hahn RT, Zamorano JL. The need for a new tricuspid regurgitation grading scheme. Eur Heart J Cardiovasc Imaging. 2017; 18:1342-3.

15. Mangieri A, Montalto C, Pagnesi M, Jabbour RJ, Rodes-Cabau J, Moat N, et al. Mechanism and implications of the tricuspid regurgitation: from the pathophysiology to the current and future therapeutic options. Circ Cardiovasc Interv. 2017;10:1730-7.

16. Khalique OK, Hahn RT. Percutaneous mitral valve repair: multi-modality cardiac imaging for patient selection and intra-procedural guidance. Front Cardiovasc Med. 2019;6:142.
17. Al-Bawardy R, Krushnaswamy A, Bhargava M, Dunn J, Wanzi O, Tuzcu M, et al Tricuspid regurgitation in patients with pacemakers and implantable cardiac defibrillators: a comprehensive review. Clin Cardiol. 2013;36:249-54.

18. Asmarats L, Dagenais F, Bédard E, Pasian S, Hahn RT, Navia JL, et al Transcatheter tricuspid valve replacement for treating severe tricuspid regurgitation: initial experience with the NaviGate bioprosthesis. Can J Cardiol. 2018;34:1370.

19. Mehr M, Taramasso M, Besler C, Ruf T, Connelly KA, Weber M, et al. 1-year outcomes after edge-to-edge valve repair for symptomatic tricuspid regurgitation: results from the TriValve Registry. ACC Cardiovasc Interv. 2019;12:1451-61.

20. Nickenig G, Kowaiski M, Hausleiter J, Braun D, Schofer J, Yzeiraj E, et al. Transcatheter treatment of severe Tricuspid regurgitation with the edge-toedge MitraClip technique. Circulation. 2017;135:1802-14.

21. Taramasso M, Alessandrini H, Latib A, Asami M, Attinger-Toller A, Biasco L, et al. Outcomes after current transcatheter tricuspid valve intervention: mid-term results from the international TriValve registry. JACC Cardiovasc Interv. 2019;12:155-65.

22. Nickenig G, Weber M, Schueler R, Hausleiter J, Näbauer M, von Bardeleben RS, et al. 6-Month outcomes of tricuspid valve reconstruction for patients with severe tricuspid regurgitation. J Am Coll Cardiol. 2019;73:1905-15.

23. Calen C, Taramasso M, Guidotti A, Kuwata S, Nietlispach F, Zuber M, et al. Successful TriCinch-in-TriCinch transcatheter tricuspid valve repair. JACC Cardiovasc Interv. 2017;10:e75-7.

24. Asmarats L, Perlman G, Praz F, Hensey M, Chrissoheris MP, Philippon F, et al. Long-term outcomes of the FORMA transcatheter tricuspid valve repair system for the treatment of severe tricuspid regurgitation: insights from the first-inhuman experience. JACC Cardiovasc Interv. 2019;12:1438-47.

25. Lauten A, Figulla HR, Unbehaun A, Fam N, Schoffer J, Doenst T, et al. Interventional treatment of severe tricuspid regurgitation: early clinical experience in a multicenter, observational, first-in-man study. Circ Cardiovasc Interv. 2018;11: e006061. 